\section{Prevalência dos defeitos de fechamento do tubo neural em recém-nascidos do Centro de Atenção à Mulher do Instituto Materno Infantil Prof. Fernando Figueira, IMIP: 2000- 2004}

\section{Neural tube defects prevalence in newborn infants in the Women Care Center of the Instituto Materno Infantil Prof. Fernando Figueira, IMIP: 2000-2004}

\author{
Sâmya Silva Pacheco 1 \\ Ariani Impieri de Souza 2 \\ Suely de Arruda Vidal 3 \\ Gláucia Virgínia de Queiroz Lins Guerra 4 \\ Malaquias Batista Filho 5 \\ Eduardo Victor de Paula Baptista 6 \\ Maria Inês Bezerra de Melo 7
}

\begin{abstract}
Objectives: to determine neural tube closing defects in newborns delivered in the maternity ward of the Women Care Center of the Instituto Materno Infantil Prof. Fernando Figueira (IMIP).

Methods: a descriptive cross-sectional cohort study, with data collected from a Born Alive Information System from which all records of newborns with fetal malformation were selected. From these records the ones pertaining to newborns between 2000 and 2004 with the neural tube closing defect were considered.

Results: of 24.964 births, 709 newborns with some type of malformation were registered. When narrowing this to the newborns with the neural tube closing defect the number disclosed was 124, with a prevalence of $0.5 \%$ of the births within the period. Newborns with the neural tube closing defect, in general, had similar characteristics to newborns with other malformations. The majority (68.5\%) of these newborns was delivered through C-Section and $37.7 \%$ had low birthweight.

Conclusions: a high prevalence of neural tube closing defect was determined, but this could have resulted from the fact that the maternity ward of Instituto Materno Infantil Prof. Fernando Figueira (IMIP) is a referral facility for high-risk pregnancies. Key words Folic acid, Neural tube defects, Information system, Live birth
\end{abstract}

\section{Resumo}

Objetivos: conhecer a prevalência de defeito de fechamento do tubo neural (DFTN) em crianças nascidas na maternidade do Centro de Atenção à Mulher do Instituto Materno Infantil Prof. Fernando Figueira (IMIP) no período de 2000 a 2004.

Métodos: estudo descritivo, do tipo corte transversal, cujos dados foram coletados de um banco de dados do Sistema de Informações sobre Nascidos Vivos do qual foram selecionados todos os registros de recémnascidos com malformação fetal. A partir daí selecionaram-se os recém-nascidos com DFTN ocorridos no período considerado.

Resultados: entre os 24.964 nascimentos, foram registrados 709 recém-nascidos apresentando alguma malformação ao nascer. Ao se considerar apenas os recém nascidos com DFTN, observaram-se 124 registros, representando uma prevalência de 0,5\% entre nascimentos naquele período. Os recém-nascidos com DFTN apresentaram características semelhantes aos recémnascidos com malformações, de uma forma geral. A maioria $(68,5 \%)$ desses recém-nascidos era de parto cesariano e 37,7\% apresentaram baixo peso ao nascer.

Conclusões: a prevalência de DFTN entre os nascimentos registrados no Centro de Atenção à Mulher do Instituto Materno Infantil Prof. Fernando Figueira foi elevada (5/1000 nascimentos) e isso, provavelmente é reflexo do fato de a maternidade ser referência para gestações de alto-risco.

Palavras-chave Acido fólico, Defeitos do tubo neural, Sistemas de informação, Nascidos vivos 


\section{Introdução}

Embora a prevalência de nascidos com anomalias congênitas nos países em desenvolvimento seja similar a dos países desenvolvidos, o impacto na saúde dos portadores desses defeitos é mais alto nos primeiros devido à falta de serviços adequados para o cuidado das crianças afetadas e ao maior índice de exposição para infecções e má nutrição materna. ${ }^{1} \mathrm{O}$ diagnóstico no pré-natal ou no parto permite a intervenção precoce, repercutindo positivamente na qualidade de vida da criança e da família. ${ }^{2}$ Por sua vez, a prevenção das malformações congênitas se faz sobretudo na saúde pública preventiva, ou seja, no nível primário. 3

Torna-se importante a identificação do tipo de malformação congênita do SNC na determinação do prognóstico, visto que os recém-nascidos com anencefalia em geral são natimortos ou morrem poucos dias após o parto, enquanto alguns casos de espinha bífida podem ser recuperados, parcial ou totalmente, com cirurgia, e outros podem até mesmo passar desapercebidos a um exame físico superficial. 4

Em 1999, o Ministério da Saúde modificou a Declaração de Nascidos Vivo (DN), do Sistema de Informações sobre Nascidos Vivos (SINASC), com a inclusão do campo 34, "Malformação congênita e/ou anomalia cromossômica", criando condições básicas para a implantação de um sistema municipal de vigilância dos defeitos congênitos. 2 Defeitos de Fechamento do Tubo Neural (DFTN), incluindo anencefalia, mielomeningocele e encefalocele, estão entre os mais comuns defeitos congênitos que afetam as taxas de mortalidade e morbidade.5As malformações secundárias ao fechamento anormal do tubo neural ocorrem entre a terceira e quarta semanas do período gestacional e, na sua etiologia, fatores genéticos e ambientais parecem estar envolvidos, 6 resultando em sua maioria, de origem multifatorial. ${ }^{7}$

A prevalência dos DFTN varia em diferentes épocas e regiões. É muito baixa na Finlândia (0,4/1000 nascimentos), alta no México (3,3/1000) e muito alta no Sul do País de Gales (até 12,5/1000). Nos Estados Unidos, situa-se em torno de 1/1000. Há ainda a possibilidade de essas freqüências estarem subestimadas quando se considera que muitas gestações são natural ou deliberadamente interrompidas. 8 São escassas as publicações no Brasil sobre a prevalência dos DFTN. Os dados disponíveis mostram taxas que variam de 0,83/1000 a 1,87/1000 nascimentos. No período de 1967 a 1995, a prevalência observada pelo Estudo Colaborativo Latino-Americano (ECLAMC) sobre $4 \mathrm{mi}$ - lhões de nascimentos na América Latina, foi de 1,5/1000.7 Os estudos de prevalência têm enorme importância prática nas políticas de saúde, visando à prevenção das anomalias congênitas; é, pois, importante conhecer as taxas antes e depois da implementação das medidas de prevenção. ${ }^{9}$ Por outro lado, os DFTN podem ter sua prevalência drasticamente diminuída se as mulheres sem antecedente de gestação afetada por DFTN consumirem $0,4 \mathrm{mg} / \mathrm{dia}$ de ácido fólico, no período peri-concepcional e as com antecedentes, utilizarem 4,0 a 5,0 mg/dia. 10

No intuito de prevenir carências nutricionais específicas de ferro e acido fólico e assim diminuir a prevalência das complicações provenientes dessas deficiências, o Ministério da Saúde do Brasil, considerando as recomendações da Organização Mundial da Saúde (OMS) e da Organização Pan-Americana da Saúde (OPAS) de fortificação de produtos alimentícios com ferro e ácido fólico, publicou a Portaria $n^{\circ} 710 / 1999$ e Resolução 344/200211,12 para regulamentar a fortificação de farinha de trigo e farinha de milho com ferro e ácido fólico.

A complicação mais referida para a deficiência de acido fólico tem sido os defeitos de fechamento do tubo neural (DFTN). Entretanto, ainda não se tem, ao nosso conhecimento, informações sobre a prevalência de DFTN entre nascidos na cidade do Recife. Além disso, o registro das informações da prevalência de DFTN ocorridos até junho de 2004 permitiria comparações futuras com a prevalência de DFTN após a implementação governamental de suplementação de ferro e folatos nos alimentos.

Diversos países têm implantado a utilização do ácido fólico, no período peri-concepcional, como medida preventiva para os defeitos do tubo neural.13 O Chile foi o primeiro país da América do Sul a adotar a suplementação do ácido fólico a alimentos e nos Estados Unidos observou-se uma redução aproximada de $19 \%$ no surgimento dessas malformações com o seu uso. 13

O objetivo do presente estudo foi conhecer a prevalência de DFTN entre as malformações de crianças nascidas na maternidade do Centro de Atenção à Mulher (CAM) do Instituto Materno Infantil Prof. Fernando Figueira (IMIP) no período de 2000 a 2004.

\section{Métodos}

O estudo foi desenvolvido na maternidade do Centro de Atenção à Mulher do Instituto Materno Infantil Prof. Fernando Figueira, em Recife, Pernambuco, Brasil, que registra uma média de 300 a 400 partos 
por mês e possui registros regulares de dados no SINASC de todos os nascimentos ocorridos na Instituição. O registro da presença de malformação foi introduzido nesse sistema a partir do ano 2000.

Foi realizado um estudo descritivo do tipo corte transversal através da coleta de informações do banco de dados do SINASC no período de 2000 a 2004. Selecionaram-se todos os registros de nascidos vivos onde havia sido anotada a presença de malformação no "campo 34" nesse banco de dados, constituindo-se um total de 709 recém-nascidos com malformação e desses 124 apresentaram DFTN.

Foram analisadas as seguintes variáveis maternas: idade, escolaridade, estado civil, duração da gestação, número de filhos vivos e/ou mortos em gravidezes anteriores, número de consultas pré-natal, ocupação, tipo de gravidez, tipo de parto e município de procedência. Em relação aos recém-nascidos foram observados o diagnóstico da malformações, o peso ao nascer e o sexo.

O estudo foi aprovado pelo Comitê de Ética e Pesquisa da Instituição e seguiu as normas estabelecidas pela resolução 196/96 da Comissão Nacional de Ética em Pesquisa do Conselho Nacional de Saúde.

\section{Resultados}

Observou-se um aumento progressivo no número de registros de malformação fetal a cada ano. No período foram registrados no banco de dados cerca de 5000 partos por ano e a freqüência de malfor-

Tabela 1

Freqüência de malformações fetais e nascimentos registrados no banco de dados do Sistema de Informações dos Nascidos Vivos (SINASC) e do Centro de Atenção à Mulher do Instituto Materno Infantil Prof. Fernando Figueira (IMIP) e proporção de malformação por nascimento por ano. Recife, Pernambuco, 2000 a 2004.

\begin{tabular}{lrrrrr}
$\begin{array}{l}\text { Ano de } \\
\text { nascimento }\end{array}$ & \multicolumn{1}{c}{ Malformações } & Nascimentos & Incidência \\
& & & & & \\
& $n$ & $\%$ & $n$ & $\%$ & $\%$ \\
\hline 2000 & 64 & 9,0 & 5393 & 21,6 & 1,19 \\
2001 & 153 & 21,6 & 4586 & 18,4 & 3,34 \\
2002 & 122 & 17,2 & 4166 & 16,7 & 2,93 \\
2003 & 178 & 25,1 & 5127 & 20,5 & 3,47 \\
2004 & 192 & 27,1 & 5692 & 22,8 & 3,37 \\
Total & 709 & 100,0 & 24.964 & 100,0 & 2,84 \\
\hline
\end{tabular}

Tabela 2

Freqüência das malformações congênitas, deformidades e anomalias cromossômicas por agrupamento segundo a Classificação Internacional de Doenças, 10 revisão (CID-10), em partos ocorridos no Centro de Atenção à Mulher do Instituto Materno Infantil Prof. Fernando Figueira (IMIP), registradas no banco de dados do Sistema de Informações dos Nascidos Vivos. Recife, Pernambuco, 2000 a 2004.

\begin{tabular}{lrr}
\hline Malformaçães & N & $\%$ \\
\hline Defeitos de fechamento do tubo neural* & 124 & 17,5 \\
Microcefalia* & 11 & 1,6 \\
Hidrocefalia* & 99 & 14,0 \\
Malformação de olho, ouvido e face & 23 & 3,2 \\
Malforamação cardíaca e aparelho circulatório & 31 & 4,4 \\
Malformação do aparelho respiratório & 29 & 4,1 \\
Malformação do trato digestivo & 14 & 2,0 \\
Malformação genital & 35 & 4,9 \\
Malformação renal & 22 & 3,1 \\
Malformação osteo-muscular & 179 & 25,3 \\
Malformações multiplas mal especificadas & 129 & 18,4 \\
Malformações cromossômicas & 13 & 1,8 \\
Total & 709 & 100,0 \\
\hline
\end{tabular}

*Malformações do sistema nervoso central $(n=234)$.

Defeitos de fechamento do tubo neural [Anecefalia $(n=24)$, Encefalocele $(n=17)$, Espinha bífida $(n=83)]$ representaram uma freqüência de 5 por 1000 nascimentos (124/24.964).

mação fetal por ano variou de $1,19 \%$ a 3,37\%, sendo a média de 2,84\% (Tabela 1 ).

As malformações do sistema nervoso central (DFTN, microcefalia e hidrocefalia) representaram $33,0 \%$ (234 casos) de todos os registros de malformados, sendo que praticamente a metade desses foram DFTN (124 casos). Em relação ao total de nascimentos (24.964) os portadores de DFTN representaram 5/1000 nascimentos (Tabela 2).

Conforme se observa na Tabela 3, 57,6\% dos recém-nascidos que nasceram com alguma malformação foram de termo.

A maioria das mães encontrava-se com idade entre 20 e 30 anos, enquanto a faixa etária de adolescentes (10 a 19 anos) representou cerca de $25,0 \%$ da amostra. Ainda na Tabela 4, em relação ao estado civil, até o ano 2003, foi observado que uma variação de $44,7 \%$ a $51,6 \%$ da amostra encontrava-se "em união consensual" e $28,1 \%$ a $33,6 \%$ se declararam casadas.

Na Tabela 5 se observa o número de filhos vivos ou mortos que a mulher declarou ter tido em gestações anteriores. Com pequenas modificações ao longo dos anos, a maioria das mulheres era primípara.

Em relação ao peso dos $\mathrm{RN}$, observou-se um elevado percentual de baixo peso ao nascer (abaixo 
Freqüência da duração da gestação em semanas de acordo com o ano de nascimento do recém-nascido com malformação registrada. Centro de Atenção à Mulher do Instituto Materno Infantil Prof. Fernando Figueira - IMIP. Recife, Pernambuco, 2000 a 2004.

\begin{tabular}{|c|c|c|c|c|c|c|c|c|c|c|c|c|}
\hline \multirow{3}{*}{ Duração da gestação } & \multicolumn{10}{|c|}{ Ano de nascimento do recém-nascido } & \multirow{2}{*}{\multicolumn{2}{|c|}{ Total }} \\
\hline & \multicolumn{2}{|c|}{2000} & \multicolumn{2}{|c|}{2001} & \multicolumn{2}{|c|}{2002} & \multicolumn{2}{|c|}{$2003 *$} & \multicolumn{2}{|c|}{2004} & & \\
\hline & $\mathrm{n}$ & $\%$ & $\mathrm{n}$ & $\%$ & $\mathrm{n}$ & $\%$ & $\mathrm{n}$ & $\%$ & $\mathrm{n}$ & $\%$ & $\mathrm{n}$ & $\%$ \\
\hline$<37$ semanas & 27 & 42,2 & 64 & 41,8 & 62 & 48,0 & 77 & 43,8 & 70 & 36,5 & 300 & 42,88 \\
\hline$\geq 37$ semanas & 37 & 57,8 & 89 & 58,2 & 60 & 52,0 & 100 & 56,2 & 122 & 63,5 & 408 & 57,6 \\
\hline Total & 64 & 100,0 & 153 & 100,0 & 122 & 100,0 & $177 *$ & 100,0 & 192 & 100,0 & 709 & 100,0 \\
\hline
\end{tabular}

* Em 2003, houve 178 registros de malformação, sendo que em um falta informação da idade gestacional.

Tabela 4

Distribuição dos recém-nascidos malformados de acordo com algumas variáveis maternas registradas no Sistema de Informações dos Nascidos Vivos - SINASC, do Instituto Materno Infantil Prof. Fernando Figueira - IMIP. Recife, Pernambuco, 2000 a 2004.

\begin{tabular}{|c|c|c|c|c|c|c|c|c|c|c|c|c|}
\hline \multirow[t]{2}{*}{ Variável materna } & \multicolumn{2}{|c|}{2000} & \multicolumn{2}{|c|}{2001} & \multicolumn{2}{|c|}{2002} & \multicolumn{2}{|c|}{2003} & \multicolumn{2}{|c|}{2004} & \multicolumn{2}{|c|}{ Total } \\
\hline & $\mathrm{n}$ & $\%$ & $\mathrm{n}$ & $\%$ & $\mathrm{n}$ & $\%$ & $\mathrm{n}$ & $\%$ & $\mathrm{n}$ & $\%$ & $\mathrm{n}$ & $\%$ \\
\hline \multicolumn{13}{|l|}{ Idade (em anos) } \\
\hline $11-19$ & 18 & 28,1 & 33 & 21,6 & 31 & 25,4 & 39 & 21,9 & 46 & 24,0 & 167 & 23,6 \\
\hline $20-25$ & 30 & 46,9 & 58 & 37,9 & 53 & 43,4 & 78 & 43,8 & 65 & 33,9 & 284 & 40,0 \\
\hline $26-30$ & 10 & 15,6 & 26 & 17,0 & 15 & 12,3 & 33 & 18,5 & 49 & 25,5 & 133 & 18,8 \\
\hline$>30$ & 6 & 9,4 & 36 & 33,5 & 23 & 18,9 & 28 & 15,8 & 32 & 16,6 & 125 & 17,6 \\
\hline \multicolumn{13}{|c|}{ Escolaridade (anos de estudo) } \\
\hline $0-3$ & 6 & 9,4 & 20 & 13,1 & 20 & 16,4 & 20 & 11,2 & 22 & 11,5 & 88 & 12,4 \\
\hline $4-7$ & 25 & 39,1 & 60 & 39,2 & 48 & 39,3 & 63 & 35,4 & 73 & 38,0 & 269 & 37,9 \\
\hline $8-11$ & 25 & 39,1 & 56 & 36,6 & 40 & 32,8 & 74 & 41,6 & 67 & 34,9 & 262 & 37,0 \\
\hline$>12$ & 8 & 12,5 & 17 & 11,1 & 14 & 11,5 & 21 & 11,8 & 30 & 15,6 & 90 & 12,7 \\
\hline \multicolumn{13}{|l|}{ Estado civil } \\
\hline Solteira & 12 & 18,8 & 28 & 18,4 & 25 & 20,5 & 45 & 25,3 & 129 & 67,2 & 239 & 33,8 \\
\hline Casada & 18 & 28,1 & 51 & 33,6 & 38 & 31,2 & 50 & 28,1 & 60 & 31,3 & 217 & 30,6 \\
\hline Viúva & 1 & 1,6 & 1 & 0,7 & 1 & 0,8 & 1 & 0,6 & 0 & 0,0 & 4 & 0,6 \\
\hline Separada & 0 & 0,0 & 4 & 2,6 & 0 & 0,0 & 2 & 1,1 & 3 & 1,6 & 9 & 1,3 \\
\hline Em união & 33 & 51,6 & 68 & 44,7 & 58 & 47,5 & 80 & 44,9 & 0 & 0,0 & 239 & 33,8 \\
\hline Total & 64 & 100,0 & 153 & 100,0 & 122 & 100,0 & 178 & 100,0 & 192 & 100,0 & 709 & 100,0 \\
\hline
\end{tabular}


Distribuição dos recém-nascidos malformados de acordo com o passado obstétrico materno registrado no Sistema de Informações dos Nascidos Vivos - SINASC, do Instituto Materno Infantil Prof. Fernando Figueira - IMIP. Recife, Pernambuco, 2000 a 2004.

\begin{tabular}{|c|c|c|c|c|c|c|c|c|c|c|c|c|}
\hline \multirow[t]{2}{*}{ Filhos tidos } & \multicolumn{2}{|c|}{2000} & \multicolumn{2}{|c|}{2001} & \multicolumn{2}{|c|}{2002} & \multicolumn{2}{|c|}{2003} & \multicolumn{2}{|c|}{2004} & \multicolumn{2}{|c|}{ Total } \\
\hline & $\mathrm{n}$ & $\%$ & $\mathrm{n}$ & $\%$ & $\mathrm{n}$ & $\%$ & $\mathrm{n}$ & $\%$ & $\mathrm{n}$ & $\%$ & $n$ & $\%$ \\
\hline \multicolumn{13}{|c|}{ Número de filhos vivos } \\
\hline 0 & 35 & 54,7 & 63 & 41,2 & 57 & 46,7 & 104 & 58,4 & 96 & 50,3 & 355 & 50,1 \\
\hline 1 & 19 & 29,7 & 55 & 35,9 & 26 & 21,3 & 31 & 17,4 & 50 & 26,2 & 181 & 25,5 \\
\hline 2 & 7 & 10,9 & 19 & 12,4 & 18 & 14,8 & 24 & 13,5 & 29 & 15,2 & 97 & 13,7 \\
\hline$>3$ & 3 & 4,7 & 16 & 10,5 & 21 & 17,2 & 19 & 0,7 & 16 & 8,4 & 75 & 10,6 \\
\hline Ignorado & 0 & 0,0 & 0 & 0,0 & 0 & 0,0 & 0 & 0,0 & 1 & 0,5 & 1 & 0,1 \\
\hline \multicolumn{13}{|c|}{ Número de filhos mortos } \\
\hline 0 & 61 & 95,3 & 134 & 87,6 & 109 & 89,3 & 168 & 94,4 & 173 & 90,1 & 645 & 91,1 \\
\hline 1 & 3 & 4,7 & 13 & 8,5 & 10 & 8,2 & 6 & 3,4 & 12 & 6,3 & 44 & 6,2 \\
\hline 2 & 0 & 0,0 & 3 & 2,0 & 2 & 1,6 & 4 & 2,2 & 2 & 1,0 & 11 & 1,6 \\
\hline$>3$ & 0 & 0,0 & 3 & 2,0 & 1 & 0,8 & 0 & 0,0 & 4 & 2,1 & 8 & 1,1 \\
\hline Ignorado & 0 & 0,0 & 0 & 0,0 & 0 & 0,0 & 0 & 0,0 & 1 & 0,5 & 1 & 0,1 \\
\hline Total & 64 & 100,0 & 153 & 100,0 & 122 & 100,0 & 178 & 100,0 & 192 & 100,0 & 709 & 100,0 \\
\hline
\end{tabular}

\section{Tabela 6}

Distribuição dos recém-nascidos malformados de acordo com o peso ao nascer registrado em cada ano e em particular aqueles com defeitos do tubo neural. Sistema de Informações dos Nascidos Vivos - SINASC, do Instituto Materno Infantil Prof. Fernando Figueira - IMIP. Recife, Pernambuco, 2000 a 2004.

\begin{tabular}{|c|c|c|c|c|c|c|c|c|c|c|c|c|}
\hline \multirow{2}{*}{$\begin{array}{l}\text { Peso do RN } \\
\text { (em gramas) }\end{array}$} & \multicolumn{2}{|c|}{2000} & \multicolumn{2}{|c|}{2001} & \multicolumn{2}{|c|}{2002} & \multicolumn{2}{|c|}{2003} & \multicolumn{2}{|c|}{2004} & \multicolumn{2}{|c|}{ Total } \\
\hline & $\mathrm{n}$ & $\%$ & $\mathrm{n}$ & $\%$ & $\mathrm{n}$ & $\%$ & $\mathrm{n}$ & $\%$ & $\mathrm{n}$ & $\%$ & $\mathrm{n}$ & $\%$ \\
\hline$<2500 \mathrm{~g}$ & 26 & 40,6 & 57 & 37,3 & 44 & 36,1 & 63 & 35,4 & 75 & 39,1 & 265 & 37,4 \\
\hline $2500-4000 \mathrm{~g}$ & 37 & 57,9 & 88 & 57,5 & 71 & 58,2 & 108 & 60,7 & 112 & 58,3 & 416 & 58,7 \\
\hline$>4000 \mathrm{~g}$ & 1 & 1,6 & 8 & 5,2 & 7 & 5,7 & 7 & 3,9 & 5 & 2,6 & 28 & 3,9 \\
\hline Total & 64 & 100,0 & 153 & 100,0 & 122 & 100,0 & 178 & 100,0 & 192 & 100,0 & 709 & 100,0 \\
\hline
\end{tabular}

Peso do recém-nascidos com defeitos de fechamento do tubo neural (em gramas)

\begin{tabular}{lrc}
\hline & $\mathrm{n}$ & $\%$ \\
\hline$<2500 \mathrm{~g}$ & 46 & 37,1 \\
$25004000 \mathrm{~g}$ & 76 & $61,3 /$ \\
$>4000 \mathrm{~g}$ & 2 & 1,6 \\
Total & 124 & 100,0 \\
\hline
\end{tabular}


de $2500 \mathrm{~g}$ ) tanto entre a população de RN malformados $(37,4 \%)$ quanto entre os RN com malformações do DFTN $(37,1 \%)$ (Tabela 6$)$.

De acordo com a Tabela 7 , comparando-se o perfil das mães dos recém-nascidos com alguma malformação ao nascer e especificando as dos com DFTN, verifica-se que eram na sua maioria procedentes do Recife, tinham ocupação do lar, realizaram quatro a seis consultas pré-natal, parto cesárea e gestação única. 54,2\% dos RN malformados, em

Tabela 7

Distribuição de variáveis maternas, sócio-demográficas e características de pré-natal registradas entre os malformados em geral e com defeitos do fechamento do tubo neural. Sistema de Informações dos Nascidos Vivos SINASC, do Instituto Materno Infantil Prof. Fernando Figueira - IMIP. Recife, Pernambuco, 2000 a 2004.

\begin{tabular}{|c|c|c|c|c|}
\hline \multirow[t]{2}{*}{ Variáveis } & \multicolumn{2}{|c|}{$\begin{array}{l}\text { Recém-nascidos } \\
\text { com malformação }\end{array}$} & \multicolumn{2}{|c|}{ DFTN } \\
\hline & $\mathrm{n}$ & $\%$ & $\mathrm{n}$ & $\%$ \\
\hline \multicolumn{5}{|c|}{ Número de consultas no pré-natal } \\
\hline Nenhuma & 33 & 4,7 & 2 & 1,6 \\
\hline 1 a 3 & 143 & 20,2 & 28 & 22,6 \\
\hline 4 a 6 & 328 & 46,2 & 54 & 43,5 \\
\hline$\geq 7$ & 98 & 27,9 & 38 & 30,6 \\
\hline Ignorado & 7 & 1,0 & 2 & 1,6 \\
\hline \multicolumn{5}{|c|}{ Ocupação da mãe } \\
\hline Do lar & 503 & 70,9 & 90 & 72,6 \\
\hline Estudante & 50 & 7,1 & 8 & 6,5 \\
\hline Outras & 156 & 22,0 & 26 & 21,0 \\
\hline \multicolumn{5}{|c|}{ Tipo de gravidez } \\
\hline Única & 684 & 96,5 & 119 & 96,0 \\
\hline Dupla & 25 & 3,5 & 5 & 4,0 \\
\hline \multicolumn{5}{|l|}{ Tipo de parto } \\
\hline Vaginal & 279 & 39,4 & 39 & 31,5 \\
\hline Cesárea & 430 & 60,6 & 85 & 68,5 \\
\hline \multicolumn{5}{|c|}{ Sexo do recém-nascido } \\
\hline Feminino & 311 & 43,9 & 65 & 52,4 \\
\hline Masculino & 384 & 54,2 & 59 & 47,6 \\
\hline Ignorado & 14 & 2,0 & 0 & 0 \\
\hline \multicolumn{5}{|l|}{ Município } \\
\hline Recife & 193 & 27,2 & 28 & 22,6 \\
\hline Jaboatão & 112 & 15,8 & 17 & 13,7 \\
\hline Olinda & 26 & 3,7 & 6 & 4,8 \\
\hline Outros & 378 & 53,3 & 73 & 58,9 \\
\hline Total & 709 & 100,0 & 124 & 100,0 \\
\hline
\end{tabular}

DFTN = Defeitos de fechamento do tubo neural. geral, do sexo masculino e $52,4 \%$ dos especificadamente com DFTN do sexo feminino.

\section{Discussão}

A proporção de $2,8 \%$ de malformações entre 24.964 nascidos vivos no período do estudo, 2000 a 2004 , foi semelhante à encontrada no Estudo Colaborativo Latino-Americano de Malformações Congênitas (ECLAMC) (2,7\%).12 Entretanto, em relação aos defeitos de fechamento do tubo neural, observou-se uma freqüência alta (5:1000 nascimentos) quando comparada a observada no ECLAMC, cuja incidência foi de 1,5: 1000, mas, próxima aos resultados encontrados por Grillo e Silva, 8 no México (3,3: 1000 nascimentos).

Em relação à hidrocefalia, contatou-se uma freqüência de 7,3\%, equivalente à encontrada pelo ECLAMC, no período de 1982 a1998 (7,4\%).14

A incidência de espinha bífida na amostra $(11,7 \%)$ foi próxima a da Irlanda do Norte $(11,2 \%)$ e do México $(10,2 \%)$, mas muito superior a do ECLAMC $(7,1 \%)$ e de países como Austrália $(7,0 \%)$, Irã $(5,4 \%)$, Inglaterra $(5,3 \%)$, Noruega $(4,7 \%)$, EUA $(4,5 \%)$, Japão $(3,7 \%)$, França $(3,3 \%)$ e Finlândia $(1,6 \%)$. Também se revelou alta quanto comparada a do Hospital dos Servidores Públicos do Estado de São Paulo, - que desenvolve o projeto ECLAMC/ Monitor - e que apresenta 5,6\%, e em relação a um estudo feito no Hospital de Clínica de Porto Alegre em 1989 (4,87\%). 4

Os achados de anencefalia $(7,3 \%)$ foram superiores à média dos hospitais do $\operatorname{ECLAMC~}(6,3 \%)$, da China $(5,4 \%)$, Austrália $(4,7 \%)$, Noruega $(4,3 \%)$, Japão (4,2\%), Finlândia (1,1\%), França $(1,0 \%)$ e do Hospital Público dos Servidores do Estado de São Paulo(3,2\%). 4

Em relação à microcefalia, a incidência mostrouse alta $(7,3 \%)$, sendo superior a da Austrália $(2,1 \%)$, Irã $(0,8 \%)$ e do Hospital de Clínicas de Porto Alegre em $1989(3,25 \%) .4$

De um modo geral, a freqüência de malformações congênitas do sistema nervoso central verificada no presente estudo foi elevada quando comparada a de países desenvolvidos. ${ }^{4,8} \mathrm{O}$ motivo que pode ter contribuído para que a maternidade do CAM/IMIP tenha tido uma alta freqüência de malformações pode estar relacionado ao fato de o hospital ser centro de referência para gestações de alto risco, fazendo com que uma grande quantidade de casos de malformações diagnosticados nesse estudo tenham sido encaminhados de outros serviços de saúde e hospitais de menor resolução. 
Algumas características comuns encontradas em países em desenvolvimento, tais como, níveis educacionais e econômicos da população baixos, alta incidência de doenças infecciosas e carênciais, escassos recurso para saúde e pesquisa, prática freqüente e sem controle de auto-medicação, facilidade de obtenção de medicações que deveriam estar submetidas à prescrição médica, qualidade ambiental precária ou mesmo condições de trabalho insalubres durante a gravidez, podem tornar o problema das malformações congênitas mais sério. 15

A distribuição da idade das mães dos recémnascidos malformados foi equivalente à encontrada na população atendida na maternidade do IMIP,16,17 não se observando nenhuma relação com a presença de malformação.

A escolaridade das mulheres também não apresentou nenhuma distribuição particular. O percentual de escolaridade abaixo de três anos de estudo (o que representa as analfabetas ditas funcionais) foi semelhante ao percentual de escolaridade acima de 12 anos de estudo, enquanto a maioria situou-se entre 4 e 11 anos de estudo. Esses percentuais também não

\section{Referências}

1. Penchaszadeh VB. Preventing congenital anomalies in developing countries. Commnity Genet. 2002; 5: 61-9.

2. Aerts D, Cunha J, Livi K, Leite JC, Flores R. Defeitos congênitos em Porto Alegre: uma estratégia para o resgate do sub-registro no SINASC. Porto Alegre: Secretaria de Vigilância em Saúde; 2004.

3. Apfel MIR, Santa Rosa AAP, Ferreira VI, Diamant L, Costa RF. Prevenção de malformações congênitas. J Bras Med. 2002; 83: 36-41.

4. Rotta NT, Aragon de Vecino MC. Malformações congênitas do sistema nervoso central: incidência de cinco anos no Hospital de Clínicas de Porto Alegre. Rev Hosp Clin Port Alegre. 1989; 9: 10-4.

5. Birnbacher R, Messerschmidt AM, Pollak AP. Diagnosis and prevention of neural tube defects. Curr Opin Urol. 2002; 12: 461-4.

6. Frey L, Hauser WA. Epidemiology of neural tube defects. Epilepsia. 2003; 44 (Suppl 3): 4-13.

7. Gelineau-van WJ, Finnell RH. Genetics of neural tube defects. Semin Pediatr Neurol. 2001; 8: 160-4.

8. Grillo E, Silva RJM. Defeitos do tubo neural e hidrocefalia congênita: por que conhecer suas prevalências? J Pediatr (Rio J) 2003; 79: 105-6.

9. Stevenson RE, Allen HP, Pai GS, Best R, Seaver LH, Dean $\mathrm{J}$, Thompson S. Decline in prevalence of neural tube defects in a high-risk region of the United States. Pediatrics. 2000; 106: 677-83. sofreram grandes variações ao longo dos anos e foram compatíveis com os encontrados nos estudos realizados entre as parturientes atendidas no IMIP, independente da situação de malformação. 16,17

Com pequenas modificações ao longo dos anos, a maioria das mulheres não possuía filhos anteriores, ou seja, estavam em suas primeiras gestações. Essa informação também é compatível com a situação atualmente apresentada pela clientela do IMIP. ${ }^{17}$ A alta prevalência de portadores de DFTN com baixo peso ao nascer, embora condizente com a literatura, ${ }^{13}$ situou-se muito acima do percentual de recém-nascidos com baixo peso para a população atendida na Instituição (que se mostra em torno de 18,7\%), 17 mesmo considerando a prematuridade.

\section{Agradecimentos}

Ao Instituto Materno Infantil Prof. Fernando Figueira, IMIP pelo suporte e ao Conselho Nacional de Desenvolvimento Científico e Tecnológico, CNPQ pela bolsa do Programa de Iniciação Científica.

10. American Academy of Pediatrics. Committee on Genetics. Folic acid for the prevention of neural tube defects. Pediatrics. 1999; 104: 325-7.

11. ANVISA (Agência Nacional de Vigilância Sanitária). Legislação em vigilância sanitária. Resolução RDC nº 344, de 13 de dezembro de 2002: aprova o regulamento técnico para a fortificação das farinhas de trigo e das farinhas de milho com ferro e ácido fólico. Diário Oficial da União [DOU] $2002 \mathrm{dez} 13$.

12. Ministério da Saúde. Portaria $n^{\circ} 710$, de 10 de junho de 1999: aprova a Política Nacional de Alimentação e Nutrição. Diário Oficial da União [DOU] 1999 jun 11.

13. Aguiar MJB, Campos AS, Aguiar RALP, Lana AMA, Magalhães RL, Babeto LT. Defeitos de fechamento do tubo neural e fatores associados em recém-nascidos vivos e natimortos. J Pediatr (Rio J) 2003; 79: 129-34.

14. Cavalcanti DP, Salomão MA. Incidência de hidrocefalia congênita e o papel no diagnóstico pré-natal. J Pediatr (Rio J) 2003; 79: 135-40.

15. Faccini LS, Leite JCL, Sanseverino MTV, Peres RM. Avaliação de teratógenos potenciais na população brasileira. Rev Cienc Saúde Coletiva 2002; 7: 65-71.

16. Souza AI, Batista Filho M, Ferreira LOC, Figueirôa JN. Efetividade de três esquemas com sulfato ferroso para tratamento de anemia em gestantes. Rev Panam Salud Públicas. 2004; 15: 313-9. 
17. Vidal SA, Arruda BKG, Vanderlei LC, Frias PG. Avaliação da série histórica dos nascidos vivos em unidade terciária de Pernambuco: 1991 a 2000. Rev Assoc Med Bras. 2005; 51: $17-22$.

Recebido em 29 de novembro de 2005.

Versão final apresentada em 20 de fevereiro 2006

Aprovado em 27 de março de 2006 